\title{
Spatially resolved studies of extragalactic jets in high redshift radio galaxies
}

\author{
Leah K. Morabito ${ }^{1}$, Adam Deller ${ }^{2}$, J. B. R. Oonk ${ }^{1,2}$, \\ Huub Röttgering ${ }^{1}$ and George Miley ${ }^{1}$ \\ ${ }^{1}$ Leiden University, P.O. Box 9513, 2300 RA Leiden, The Netherlands \\ email: morabito@strw.leidenuniv.nl \\ ${ }^{2}$ Netherlands Institute for Radio Astronomy (ASTRON), \\ Postbus 2, 7990 AA Dwingeloo, The Netherlands
}

\begin{abstract}
The correlation between radio spectral steepness and redshift has been successfully used to find high redshift $(z \geqslant 2)$ radio galaxies, but the origin of this relation is unknown. The ultra-steep spectra of high-z radio sources make them ideally suited for studies with the Low Band Antenna of the new Low Frequency Array, which covers 10-80 MHz and has baselines up to about $1300 \mathrm{~km}$. As part of an ongoing survey, we use the longest baselines to map the lowfrequency $(<70 \mathrm{MHz})$ spatial distributions along the jets of 5 bright extended steep spectrum high-z radio sources. From this, we will determine whether the spectra change over these spatially resolved sources, thereby constraining particle acceleration processes. We present early results from our low-frequency survey of ultra-steep spectrum radio galaxies. The first low frequency long baseline images of these objects are presented.
\end{abstract}

Keywords. galaxies: high-redshift, galaxies: jets

\section{Introduction}

High redshift radio galaxies (HzRGs) are distant galaxies $(z \geqslant 2)$ with radio luminosities at $500 \mathrm{MHz}$ (rest frame) in excess of $10^{27} \mathrm{~W} \mathrm{~Hz}^{-1}$ (see review by Miley \& De Breuck 2008). They tend to be associated with steep-spectrum extended radio sources, with jets of sizes in excess of a few kiloparsec. They are often found embedded in huge ionized halos (e.g., Kurk et al. 2000), and have optical-infrared continuum sources outside of the host galaxy that are aligned with the radio jet axis (e.g., Best et al. 1996). Additionally, the more distant a radio galaxy is, the steeper its radio spectra (e.g., Tielens et al. 1979; Blumenthal \& Miley 1979, see Figure 1). This correlation between spectral index and redshift has been used to hunt for HzRGs, although the underlying cause of the correlation is not understood.

Several different theories (summarized in Miley \& De Breuck 2008) have been proposed as to why HzRGs have ultra steep spectra (USS; $\alpha>1$, where $S_{\nu} \propto \nu^{-\alpha}$ ). The conventional explanation is that a concave spectrum coupled with a radio $K$-correction produces the observed $z-\alpha$ relation. While it has been shown that the correlation weakens when shifting to the rest frame (van Breugel \& McCarthy 1990), it is still present. An alternative is that the $z-\alpha$ relation actually stems from a luminosity, $L-\alpha$ relation (Chambers et al. 1990; Blundell \& Rawlings 1999). For a flux-limited sample, the more distant observed objects will naturally be more luminous due to Malmquist bias. However, when taking objects with a limited range of $L$, Athreya \& Kapahi (1998) showed that the $z-\alpha$ relation still persists. Another possibility is that the spectral index is linked to ambient density around HzRGs, which increases with redshift (Athreya \& Kapahi 1998). An environmental explanation that relies entirely on the ambient density would have to explain why the internal lobe-to-lobe variation of spectral index is smaller than 


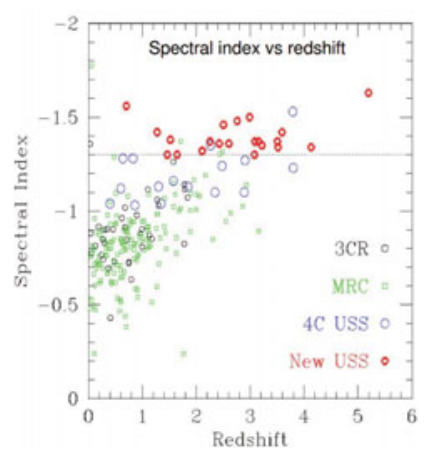

Figure 1. Spectral index vs. redshift for four different samples. Plot from De Breuck et al. (2000).

the variation between objects (Carilli et al. 1994). If the $z-\alpha$ relation is intrinsic to how the jets are generated within the HzRGs, the question then is what the particle acceleration processes are that produce the ultra steep spectra. Characterizing the spatially resolved spectral energy distributions at low frequencies is likely to help us understand the particle acceleration mechanisms and therefore the $z-\alpha$ relation. The only instrument currently capable of carrying out the required low-frequency, high resolution observations is the Low Frequency Array (LOFAR; van Haarlem et al. 2013).

\section{The Low Frequency Array}

LOFAR currently consists of 37 stations spread throughout the Netherlands, and 8 international stations throughout Europe. There are 24 'core' stations located with the central $2 \mathrm{~km}$ of the array, and 13 'remote' stations throughout the rest of the Netherlands, with baselines up to $90 \mathrm{~km}$. There are 8 international stations that provide baselines up to $1296 \mathrm{~km}$. Each station consists of a high band antenna (HBA; 120-240 MHz) and low band antenna (LBA; $10-80 \mathrm{MHz}$ ). This project uses the LBA. Each LBA station consists of 96 dual-polarization dipole antennas. Core and remote stations have diameters of 81.34 $\mathrm{m}$, and international stations have diameters of $65 \mathrm{~m}$. Only 48 antennas may be used at one time for core and remote stations, and we used the outermost 48 antennas for these stations. This configuration is known as LBA_OUTER and mitigates problems with mutual coupling due to closely spaced antennas. International stations use all 96 stations.

One of the most exciting prospects of LOFAR is that with the long baselines to the international stations it is theoretically possible to achieve sub-arcsec resolution at $60 \mathrm{MHz}$. However, making high resolution maps with LOFAR's LBA is challenging. The $u-v$ coverage is sparse, as there are large gaps between the numerous Dutch and few international stations. Correcting for the ionosphere is a challenge, as it can have a large impact on the visibilities at low frequencies. This is exacerbated by the wide separation between the international stations, as the ionospheric conditions seen by one station can be vastly different to those seen by another station. If the target source itself is not bright enough to provide the necessary calibration solutions, a very nearby calibrator source is needed, and a sufficiently dense calibrator catalogue for International LOFAR observations does not yet exist (although work is underway; Moldon et al. 2014, submitted). Despite these issues, early work has shown that long baseline fringes and images are possible with just a handful of international and core stations (Wucknitz 2010).

\section{The ultra steep spectrum survey}

We started with a catalogue of HzRGs in the northern hemisphere with known ultra steep spectra (Miley \& De Breuck 2008). We used the spectral indices to predict fluxes 
at $30 \mathrm{MHz}$ and selected objects with an expected flux at $30 \mathrm{MHz}$ above $10 \mathrm{Jy}$. Compact objects with largest angular size less than 5 arcsec were discarded, as well as objects exhibiting very disturbed extended structure that would be difficult to image. The resulting sample (listed in the table below) consists of ten of the brightest HzRGs in the Northern Hemisphere, with the addition of 3C 368, a low-redshift analogue.

\begin{tabular}{lcccccc}
\hline Source & $\mathbf{J 2 0 0 0}$ & $\mathbf{z}$ & $\begin{array}{c}\mathbf{S}_{\mathbf{1 7 8}} \\
{[\mathbf{J y}]}\end{array}$ & $\boldsymbol{\alpha}_{\mathbf{1 7 8}}^{\mathbf{1 4 0 0}}$ & $\begin{array}{c}\mathbf{S}_{\mathbf{3 0}} \\
{[\mathbf{J y}]}\end{array}$ & $\mathbf{L A S}$ \\
\hline 4C 41.17 & $0650+4130$ & 3.792 & 2.7 & -1.12 & 20 & $13^{\prime \prime}$ \\
3C 9 & $0020+1540$ & 2.02 & 19.4 & -1.06 & 128 & $9.6^{\prime \prime}$ \\
3C 368 & $1805+1101$ & 1.131 & 15 & -1.2 & 127 & $11^{\prime \prime}$ \\
4C 39.37 & $1235+3925$ & 3.221 & 5.3 & -1.47 & 73 & $8^{\prime \prime}$ \\
4C 60.07 & $0512+6030$ & 3.791 & 3.1 & -1.45 & 41 & $9^{\prime \prime}$ \\
4C 72.26 & $1908+7220$ & 3.537 & 3.2 & -1.15 & 23 & $15.4^{\prime \prime}$ \\
4C 28.58 & $2351+2910$ & 2.905 & 2.9 & -1.17 & 23 & $14.5^{\prime \prime}$ \\
4C 24.28 & $1348+2415$ & 2.889 & 4.8 & -1.05 & 31 & $6^{\prime \prime}$ \\
4C 43.15 & $0735+4344$ & 2.429 & 4.5 & -0.95 & 21 & $10.8^{\prime \prime}$ \\
4C 48.48 & $1933+4811$ & 2.343 & 3 & -1.02 & 18 & $14^{\prime \prime}$ \\
4C 19.71 & $2144+1929$ & 3.592 & 2.5 & -0.96 & 14 & $8.9^{\prime \prime}$ \\
\hline
\end{tabular}

Table 1. Some properties of the $10(+1$ low- $z$ analogue) USS objects in the survey.

We began observing targets during Cycle 0 of LOFAR operations (Sep 2013) and are observing 2-3 targets per every 6 months, based on target availability during night-time, to mitigate effects from the ionosphere. At the time of writing, we had observed 6 targets. Each target is observed for either 8.5 or $2 \times 6 \mathrm{hr}$, with continuous frequency coverage from 30 to $78 \mathrm{MHz}$. We use the multibeam capability of LFOAR to simultaneously observe a calibrator with identical frequency and time coverage. The survey is expected to be complete in mid-2015.

\section{Data reduction}

All data were pre-processed by the Radio Observatory, including RFI excision. We flagged non-functioning stations and times where the data were not coherent. Normally, $2 \%$ to $10 \%$ of the data was flagged. We subtracted the bright, distant sources that contributed significantly to the measured signal, using the "demixing" algorithm developed by Sebastian van der Tol. The data reduction was further divided into two parts: calibration of the Dutch stations only, and calibration of the long baselines.

Calibrator solutions for the Dutch array were calculated using the BlackBoard Selfcal software system (Pandey et al. 2009) and transferred to the target. We performed a phase-only self-calibration for the Dutch array only on the target field using a sky model built from the NVSS and WENSS surveys. Low resolution images ( $\sim 11$ arcsec beam) of the targets were made using about $2 \mathrm{MHz}$ of bandwidth, and the integrated flux densities were extracted using the CASA tool imfit. The core stations are all on a single clock, and further delay calibrations are unnecessary amongst these stations. We therefore combine all core stations into one large 'super' station for the calibration of the longer baselines.

At this point the data were converted to circular polarization, written into uvfits format, and read into AIPS. We used a nominal amplitude correction (which combines pre-correlator voltage scaling at the stations, correlator effects and the nominal station SEFD) that was derived empirically based on a number of observations and is thought to 


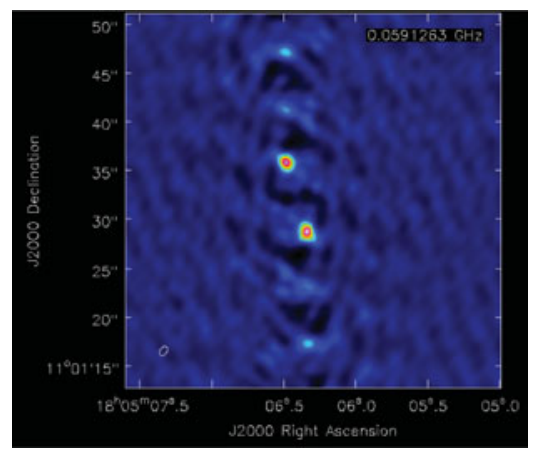

Figure 2. Initial image of $3 \mathrm{C} 368$. Image noise (away from beam artifacts) is $192 \mathrm{mJy}$. The restoring beam is $1.1 \times 0.9$ arcsec.

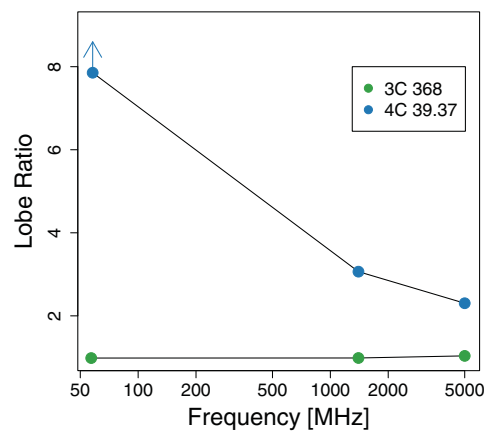

Figure 3. Lobe ratios (defined as North:South) for two fringe-fitted objects. The arrow denotes an upper limit.

be correct to within a factor of several. The flux scale was set to the total integrated flux measured from the low-resolution images. We used higher frequency models to perform fringe-fitting, excluding the remote stations to first calibrate the international stations. The high frequency models come from VLA L-band, A-Array images, which have restoring beams of about 1.4 arcsec. We imaged the fringe-fitted data, and then self-calibrated to further correct the amplitudes on the international stations. We are also investigating how much the resulting images depend on the starting model for the fringe-fitting.

\section{Early results and future work}

Figure 2 shows an initial image of $3 \mathrm{C} 368$. The noise is estimated from the expected flux as $192 \mathrm{mJy}$, still at least an order of magnitude larger than desired. The artifacts in the image in the North-South direction come from incomplete $u-v$ coverage. The restoring beam is $1.1 \times 0.9$ arcsec, and shows that images can be made with arcsec resolution at $60 \mathrm{MHz}$.

We can now begin to study the internal properties of the HzRGs. The lobe-to-lobe flux ratio is of particular interest, as there are claims (Carilli et al. 1994) that the internal, lobe-to-lobe, variation of spectral index is smaller than the variation between sources. This would indicate that the spectral index is likely more dependent on an internal rather than an external mechanism. We have plotted this ratio against frequency for two of the sources in Figure 2. The lobe ratio for 3C 368 stays constant over three orders of magnitude, while the lobe ratio for $4 \mathrm{C} 39.37$ changes dramatically. It is encouraging for our fringe fitting method, which requires an input model, that even when we provide a 
starting model of two point sources, 4C 39.37 only has one lobe detected at $60 \mathrm{MHz}$. In this case, at least, the fringe fitting is model independent.

Future work includes improving the calibration of the data we have, and processing the remaining targets. The observations for the survey are expected to be finished mid2015 , and will provide important information to understand the origin of the ultra steep spectra of HzRGs, and ultimately the $z-\alpha$ relation.

\section{Acknowledgements}

LKM acknowledges financial support from NWO Top LOFAR project, project No. 614.001.006.

\section{References}

Athreya, R. M. \& Kapahi, V. K. 1998, JA\&A, 19, 63

Best, P. N., Longair, M. S., \& Rottgering, H. J. A. 1996, MNRAS, 280, L9

Blumenthal, G. \& Miley, G. 1979, A\&A, 80, 13

Blundell, K. M. \& Rawlings, S. 1999, Nature, 399, 330

Carilli, C. L., Owen, F. N., \& Harris, D. E. 1994, AJ, 107, 480

Chambers, K. C., Miley, G. K., \& van Breugel, W. J. M. 1990, ApJ, 363, 21

De Breuck, C., van Breugel, W., Röttgering, H. J. A., \& Miley, G. 2000, A\& $A S$, 143, 303

Kurk, J. D., Röttgering, H. J. A., Pentericci, L., et al. 2000, A\&AA, 358, L1

Miley, G. \& De Breuck, C. 2008, A\&AR, 15, 67

Pandey, V. N., van Zwieten, J. E., de Bruyn, A. G., \& Nijboer, R. 2009, The Low-Frequency Radio Universe, 407, 384

Tielens, A. G. G. M., Miley, G. K., \& Willis, A. G. 1979, A\& AS, 35, 153

van Breugel, W. J. M. \& McCarthy, P. J. 1990, Evolution of the Universe of Galaxies, 10, 359

van Haarlem, M. P., Wise, M. W., Gunst, A. W., et al. 2013, A\& A, 556, A2

Wucknitz, O. 2010, ISKAF2010 Science Meeting 